\section{\$12. Two-dimensional Spatial Temperature and Density Measurements in an Arcjet Plasma Expanding through a Slit Nozzle}

Namba, S., Kozue, K., Endo, T., Takiyama, K. (Grad. School Eng. Hiroshima Univ.),

Sato, K., Tamura, N.

Arcjet plasmas have been applied in various scientific and engineering fields such as material processing (welding and cutting), chemical vapor deposition (CVD), waste treatment, and plasma propulsion. For example, arcjet thrusters with a power range from several hundred watts to $100 \mathrm{~kW}$ have been developed for the main engine system of a spacecraft and the orbital and attitude control of a small satellite.

We have developed various arcjet plasma sources with axially symmetric structures [1]. High-density helium plasma expanding through an axisymmetric converging diverging nozzle was observed by using a visible spectrometer to estimate the plasma parameters. An analysis of the continuum and line spectra showed that the electron temperature and density of the expanding plasma were 0.3 $\mathrm{eV}$ and $2.0 \times 10^{15} \mathrm{~cm}^{-3}$, respectively, at a position $45 \mathrm{~mm}$ from the nozzle exit. However, in an axisymmetric device, the arc discharging section in which the thermal arc plasma is generated cannot be observed directly. Therefore, the temperature, density, and flow of the arcjet plasmas inside the nozzle are not well understood.

In order to measure the plasma parameters, we developed an arcjet plasma device having a rectangular converging and diverging slit nozzle [2]. A pair of molybdenum anodes served as the slit nozzle; the separation between them was $0.5 \mathrm{~mm}$. The cathode was a needle-shaped rod of cerium tungsten. The throat width and length were 10.0 and $1.0 \mathrm{~mm}$, respectively, and the diverging angle was $30^{\circ}$. An atmospheric thermal arc plasma was generated between the anode nozzle and the cathode. The gap length between the anode and cathode was around 1.0 $\mathrm{mm}$. In this study, the discharge current and its voltage were $20 \mathrm{~A}$ and $\sim 20 \mathrm{~V}$, respectively, and the helium gas pressure was about $100 \mathrm{kPa}$ in the discharge region. A visible spectrometer with a focal length of $0.5 \mathrm{~m}$ was used to examine the characteristics of the expanding arcjet plasma.

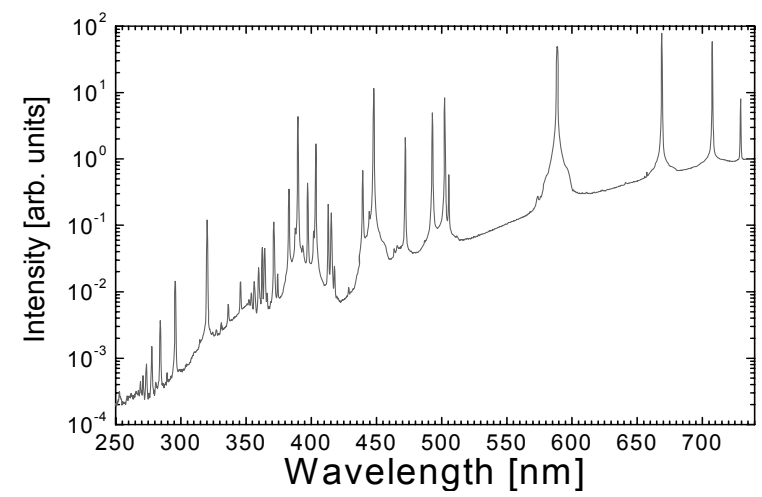

Fig.1. Typical emission spectrum emitted from He arcjet.
The diffraction gratings were 150 grooves $/ \mathrm{mm}$ and 1200 grooves $/ \mathrm{mm}$. The detector was a charge-coupled device camera. The 2D distribution of the plasma emission was obtained by moving the focus position onto the fiber by employing an optical stage. When the emission at wavelengths above $400 \mathrm{~nm}$ was measured, a sharp cut filter was used to block the second- and third-order spectra.

Figure 1 shows typical emission spectrum observed at 2 $\mathrm{mm}$ downstream from the throat. As clearly seen, intense continuum spectra as well as line emission attributed to He I transitions were observed. The continuum spectra probably originated from bremsstrahlung radiation in visible wavelength region and from the radiative recombination in UV region. From an analysis of the continuum emissions, we derived the two dimensional electron temperature distribution, as shown in FIG. 2 (a). The highest temperature inside the nozzle was approximately $6,000 \mathrm{~K}$ [3]. On the other hand, the electron density was determined by Stark broadening width relevant to He I 667.8-nm spectrum. Figure 2 (b) represents the 2D spatial distribution of the density. The density in the nozzle reached $\sim 2.7 \times 10^{15} \mathrm{~cm}^{-3}$ [3].

(a)

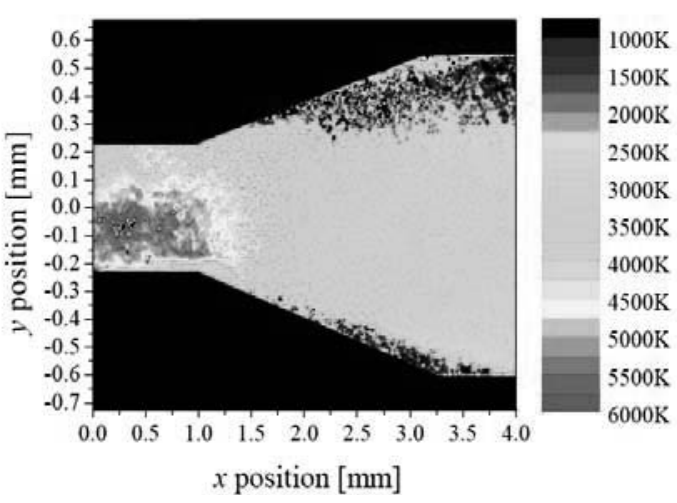

(b)

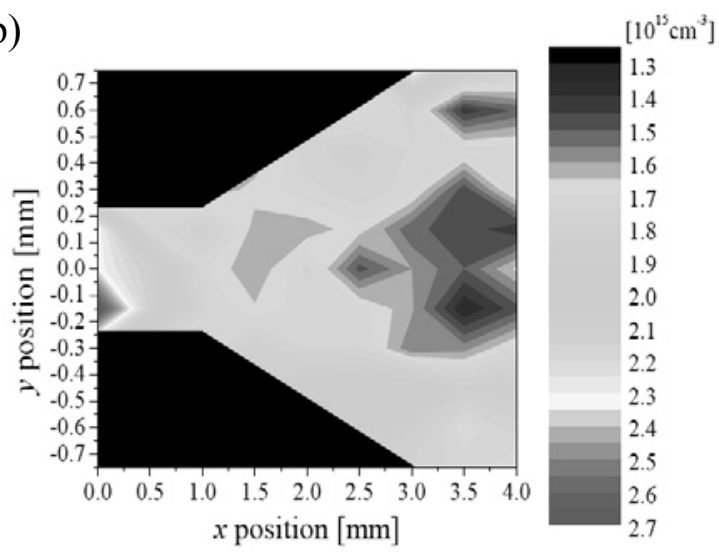

Fig. 2. 2D electron temperature (a) and density (b) distributions inside the slit nozzle.

[1] Namba, S., et al., J. Plasma and Fusion Res. Series, 8, 1348 (2009)

[2] Namba, S., et al., Jpn. J. Appl. Phys. 48, 116005 (2009).

[3] Kozue, K., et al., Plasma and Fusion Research, 6, 2406054 (2010). 